International Conference Mathematical and Computational Biology 2011

International Journal of Modern Physics: Conference Series

Vol. 9 (2012) 456-463

C World Scientific Publishing Company

DOI: $10.1142 / \mathrm{S} 2010194512005545$

\title{
NATURE-INSPIRED COGNITIVE EVOLUTION TO PLAY MS. PAC-MAN
}

\author{
TSE GUAN TAN \\ Evolutionary Computing Laboratory, \\ School of Engineering and Information Technology, Universiti Malaysia Sabah, \\ 88400, Kota Kinabalu, Sabah, Malaysia \\ tseguantan@gmail.com \\ JASON TEO \\ Evolutionary Computing Laboratory, \\ School of Engineering and Information Technology, Universiti Malaysia Sabah, \\ 88400, Kota Kinabalu, Sabah, Malaysia \\ jtwteo@ums.edu.my \\ PATRICIA ANTHONY \\ Center of Excellence in Semantic Agents, \\ Universiti Malaysia Sabah \\ 88400, Kota Kinabalu, Sabah, Malaysia \\ panthony@ums.edu.my
}

\begin{abstract}
Recent developments in nature-inspired computation have heightened the need for research into the three main areas of scientific, engineering and industrial applications. Some approaches have reported that it is able to solve dynamic problems and very useful for improving the performance of various complex systems. So far however, there has been little discussion about the effectiveness of the application of these models to computer and video games in particular. The focus of this research is to explore the hybridization of nature-inspired computation methods for optimization of neural network-based cognition in video games, in this case the combination of a neural network with an evolutionary algorithm. In essence, a neural network is an attempt to mimic the extremely complex human brain system, which is building an artificial brain that is able to self-learn intelligently. On the other hand, an evolutionary algorithm is to simulate the biological evolutionary processes that evolve potential solutions in order to solve the problems or tasks by applying the genetic operators such as crossover, mutation and selection into the solutions. This paper investigates the abilities of Evolution Strategies (ES) to evolve feed-forward artificial neural network's internal parameters (i.e. weight and bias values) for automatically generating Ms. Pac-man controllers. The main objective of this game is to clear a maze of dots while avoiding the ghosts and to achieve the highest possible score. The experimental results show that an ES-based system can be successfully applied to automatically generate artificial intelligence for a complex, dynamic and highly stochastic video game environment.
\end{abstract}

Keywords: Evolution Strategies; evolutionary artificial neural network; Ms. Pac-man. 


\section{Introduction}

Evolutionary Algorithms (EAs) [8] as shown in Fig. 1 is a randomized heuristic for search and optimization procedures that are based on the genetic processes of biological organisms. This algorithm employs a population of individuals that encode a problem's parameters rather than a sole individual, which differs from other traditional optimization techniques such as linear programming and nonlinear programming. By working with a set of individuals, EAs are able to identify and choose promising individuals from the population. Each iteration of an EA involves four essential genetic operations: parent selection, crossover (recombination), mutation and survivor selection. During survivor selection, the worse individuals (solutions) will normally be discarded from the population. In contrast, the better individuals are recombined with other individuals to form a set of new individuals. After that, the newly created individuals will then be mutated. The crossover and mutation will alter those chosen individuals, producing potentially better individuals.

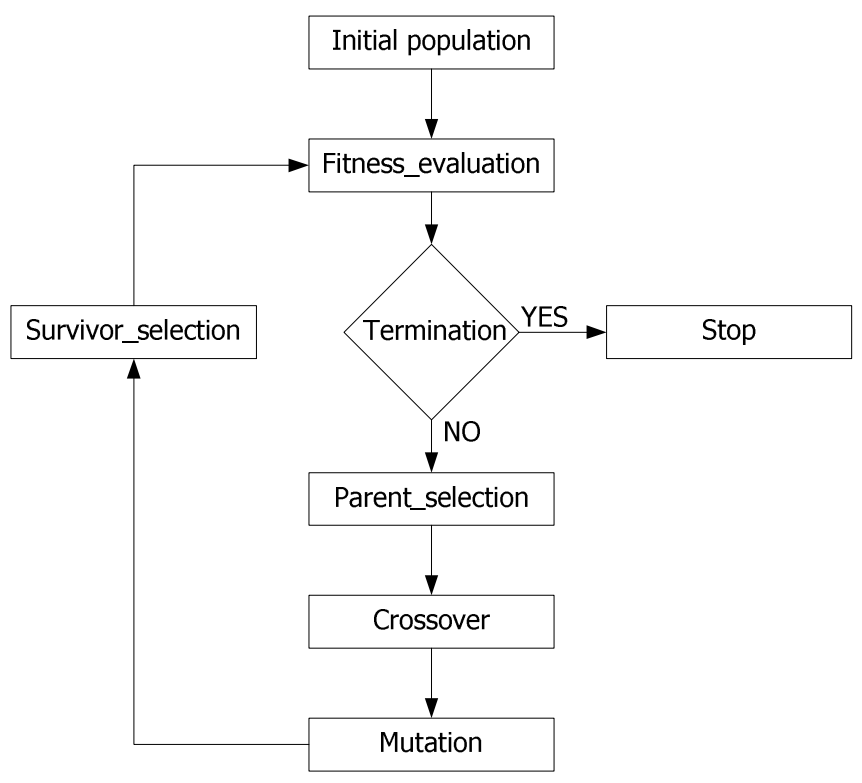

Fig. 1. Flowchart of evolutionary algorithms.

We used a hybrid method of evolution strategies (ES) together with a 2-layer feedforward artificial neural network (ANN) to generate the game controller to play a variation of the well-known Pac-man video game called Ms. Pac-man. The proposed system is called Evolution Strategies Neural Network (ESNet). This proposed algorithm will be benchmarked against Random Neural Network (RandNet) by using the international open computer game competition, Ms. Pac-man [1, 2, 3, 4]. Ms. Pac-man is a non-deterministic variation of the original Pac-man where the movements of the ghosts are unpredictable and this becomes a good challenge for computational intelligence methods to learn and play the game. Fig. 2 shows the game-play of this game. 


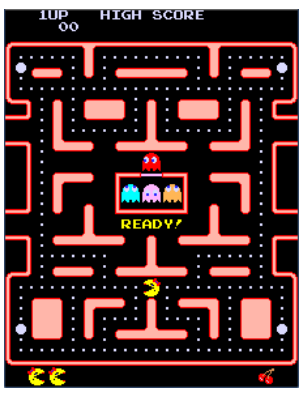

a. The first maze of Ms. Pac-man. Start the new game.

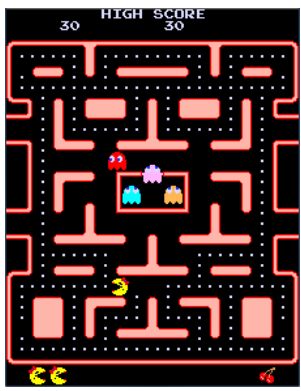

b. Agent moves around the maze to eat the pills.

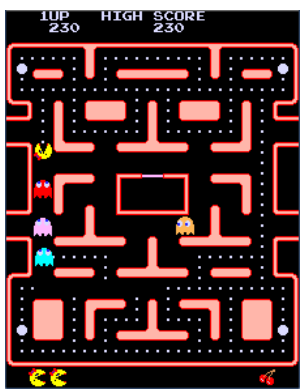

c. The ghosts chase the agent around the maze.

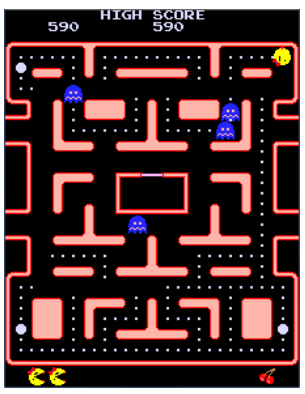

d. Agent eats a power pill and then the ghosts turn into edible ghosts.

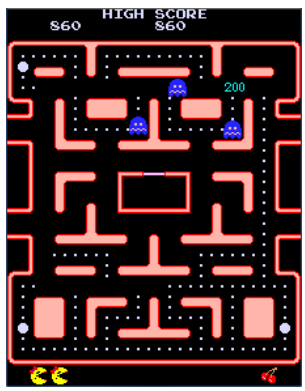

e. Agent with the temporary ability to eat the edible ghosts to increase the game score.

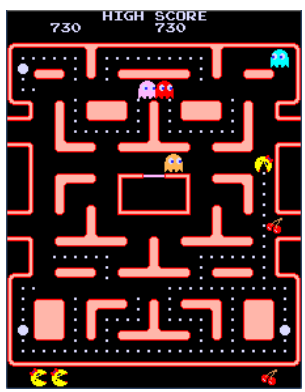

f. A fruit or pretzel appears randomly from one of the escape tunnels.

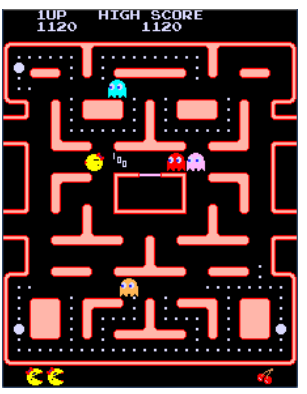

g. Eat the fruit or pretzel to earn bonus points.

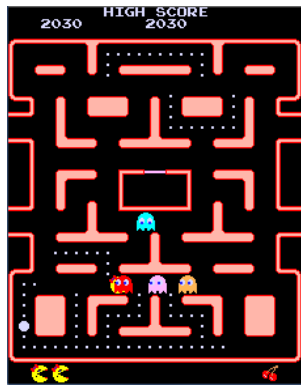

h. A ghost kills the agent, a life is lost. The game is over once all of agent's lives are lost.

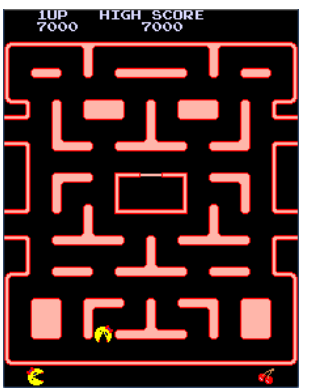

i. A level or board is completed when all pills are eaten.

Fig. 2. Ms. Pac-man game-play. 
This paper is organized as follow: in Section 2, details of the experimental algorithms and parameter setup are presented. Section 3 discusses the results. Finally, conclusions are given in the last section.

\section{Experimental Algorithms and Parameter Setting}

The structure of the RandNet controller is shown in Fig. 3. The initial connection weights and biases are set by sampling a uniform distribution with $\min =-1$ and $\max =1$, whose output is then used to control the Ms. Pac-man agent.

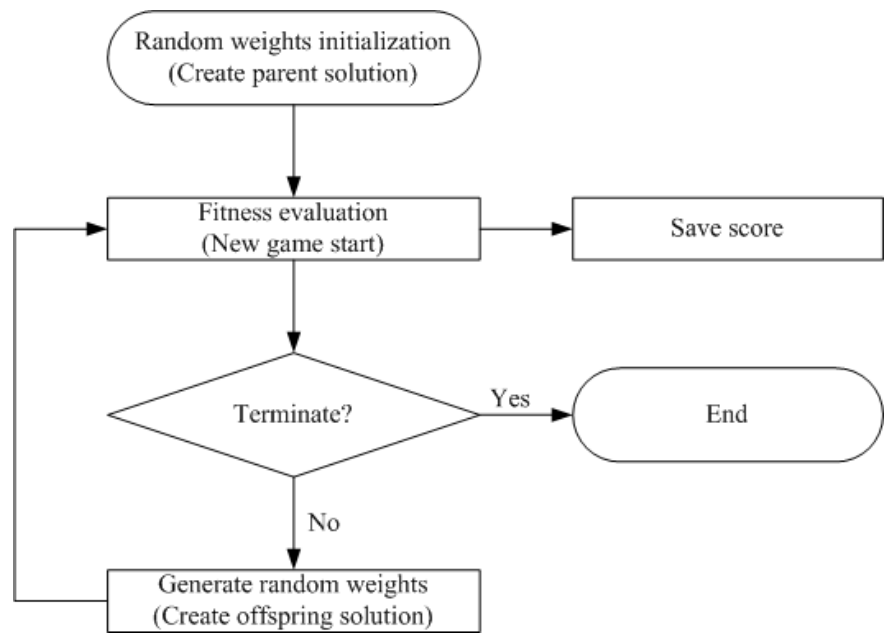

Fig. 3. The flowchart of RandNet algorithm.

The (1+1)-ES for a two membered ES has been applied to train the ANN by evolving the weights and biases, which we called the ESNet. In the initialization phase, the ANN weights and biases are encoded into a chromosome from uniform distribution with range $[-1,1]$ to act as parent and its fitness is evaluated. Subsequently, polynomial mutation operator $[6,7]$ is used with distribution index $=20.0$ to create an offspring from the parent and its fitness is evaluated. After that, the fitness of the offspring and parent are compared. If the offspring performs better than the parent, then the parent is replaced by the offspring as a new parent for the next evaluation. Otherwise the offspring is eliminated and a new mutated offspring is generated. If the parent and the offspring are incomparable, the offspring is compared with set of previously non-dominated individuals in the archive. Fig. 4 shows the flowchart of ESNet. The general parameters are summarized in Table 1. The fitness function $F$ chosen for maximization is based on the score obtained in each evaluation as follows:

$$
F=\sum_{n=1}^{N} \text { (Ms.Pacman Scores) }
$$

where $n$ and $N$ represent the number of lives in a full game. 


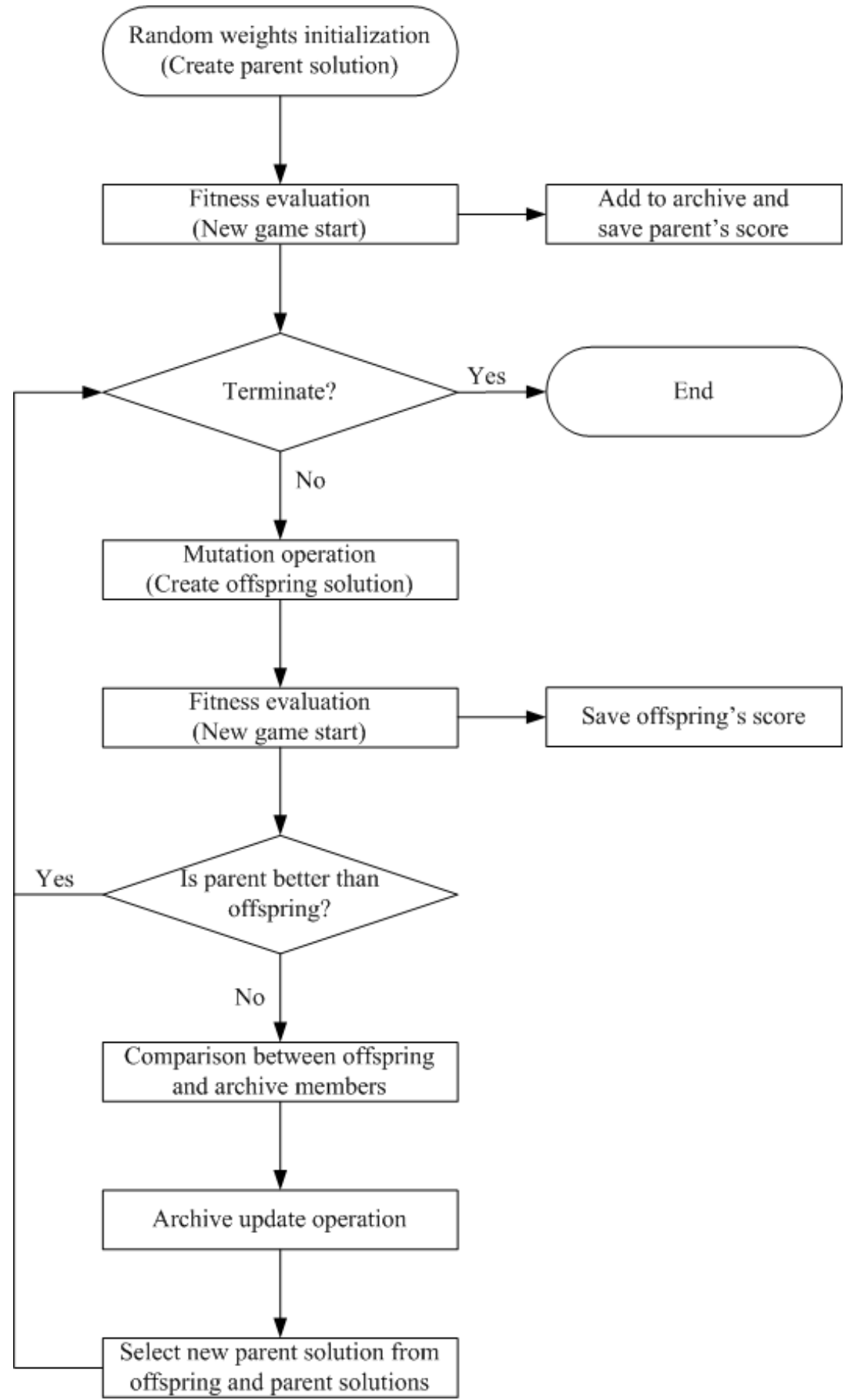

Fig. 4. The flowchart of ESNet algorithm. 
Table 1. Parameters setting.

\begin{tabular}{ll}
\hline Parameters & Setting \\
\hline Number of inputs & 5 \\
Number of outputs & 1 \\
Number of hidden layers & 1 \\
Number of hidden neurons & 20 \\
Activation function & Log-sigmoid \\
Mutation operator & Polynomial mutation \\
Mutation probability & 0.7 \\
Distribution index & 20.0 \\
Number of runs & 10 \\
Number of evaluations / run & 500 \\
\hline
\end{tabular}

\section{Results and Discussions}

Table 2 compares the game scores obtained from the RandNet and ESNet in 10 runs. As can be seen from the table, ESNet was shown to have a better performance over RandNet. It is interesting to note that the difference between the higher score of RandNet (900) and the higher score of ESNet (7430) is large. On average, the ESNet (7161) reported significantly more than the RandNet (557). The average score improved almost 6600 points from the RandNet controller. The reason is that RandNet is made randomly without any learning effort. However, the results of this study show that the evolutionary approach helps to learn and enhance the performance of the neural network.

Table 2. Game scores obtained from the RandNet and ESNet.

\begin{tabular}{llll}
\hline Run & RandNet & ESNet & Difference \\
\hline 1 & 700 & 6710 & 6010 \\
2 & 720 & 7050 & 6330 \\
3 & 670 & 7290 & 6620 \\
4 & 260 & 7190 & 6930 \\
5 & 200 & 7430 & 7230 \\
6 & 650 & 6930 & 6280 \\
7 & 420 & 7080 & 6660 \\
8 & 560 & 7280 & 6720 \\
9 & 900 & 7320 & 6420 \\
10 & 490 & 7330 & 6840 \\
Min & $\mathbf{2 0 0}$ & $\mathbf{6 7 1 0}$ & $\mathbf{6 0 1 0}$ \\
Max & $\mathbf{9 0 0}$ & $\mathbf{7 4 3 0}$ & $\mathbf{7 2 3 0}$ \\
Mean & $\mathbf{5 5 7}$ & $\mathbf{7 1 6 1}$ & $\mathbf{6 6 0 4}$ \\
\hline
\end{tabular}

A paired t-test was performed to determine if the ESNet was effective. The results of the $\mathrm{t}$-test can be seen in the resultant Table 3. The value of $\mathrm{t}(9)=-58.7441$, which can round off to -58.74 , two-tail $\mathrm{p}=6.05 \mathrm{E}-13, \mathrm{p}<0.05$ providing evidence that the ESNet is 
effective in producing better controller to play the Ms. Pac-man agent. Using the Confidence Level (95\%) value of 254.31 in the Table 4, the confidence interval is the mean plus or minus this value. Thus, a 95\% C.I. about Mean Difference is (6349.69, 6858.31).

Table 3. T-test: paired two sample for means.

\begin{tabular}{lll}
\hline & RandNet & ESNet \\
\hline Mean & 557 & 7161 \\
Variance & 47223.33 & 48165.56 \\
Observations & 10 & 10 \\
Pearson correlation & -0.32493 & \\
Hypothesized mean difference & 0 & \\
df & 9 & \\
$\mathbf{t}$ stat & $\mathbf{- 5 8 . 7 4 4 1}$ & \\
$\mathrm{P}(\mathrm{T}<=t)$ one-tail & $3.02 \mathrm{E}-13$ & \\
t Critical one-tail & 1.833113 & \\
$\mathbf{P}(\mathbf{T}<=t)$ two-tail & $\mathbf{6 . 0 5 E}-13$ & \\
t Critical two-tail & 2.262157 & \\
\hline
\end{tabular}

Table 4. The results on the difference between RandNet and ESNet.

\begin{tabular}{ll}
\hline Mean & $\mathbf{6 6 0 4}$ \\
Standard error & 112.4198 \\
Median & 6640 \\
Mode & $\#$ N/A \\
Standard deviation & 355.5028 \\
Sample variance & 126382.2 \\
Kurtosis & -0.09717 \\
Skewness & 0.075576 \\
Range & 1220 \\
Minimum & 6010 \\
Maximum & 7230 \\
Sum & 66040 \\
Count & 10 \\
Confidence level $\mathbf{( 9 5 . 0 \% )}$ & $\mathbf{2 5 4 . 3 1 1 4}$ \\
\hline
\end{tabular}

\section{Conclusions}

The aim of this paper was to develop a high-scoring agent by using an evolutionary algorithm. We presented evolution strategies to evolve the neural network, and compared it to a random system for playing Ms. Pac-man. The results show that the ESNet performed very well compared to RandNet. The evolution strategies displayed a remarkable ability to evolve sophisticated and effective neural network in a nondeterministic and dynamic environment. 


\section{Acknowledgments}

This research is funded under the ScienceFund project SCF52-ICT-3/2008 granted by the Ministry of Science, Technology and Innovation, Malaysia.

\section{References}

1. IEEE Congress on Evolutionary Computation (CEC) Competition Homepage (2007), http://cec2007.nus.edu.sg/staticPages/Competitions.html, accessed on September 27, 2008.

2. IEEE Congress on Evolutionary Computation (CEC) Competition Homepage (2009), http://www.cec-2009.org/competitions.shtml, accessed on October 10, 2009.

3. IEEE Symposium on Computational Intelligence and Games (CIG) Competition Homepage (2009), http://www.ieee-cig.org/cig-2009/competitions/, accessed on October 21, 2009.

4. IEEE World Congress on Computational Intelligence (WCCI) Competition Homepage (2008), http://www2.mae.cuhk.edu.hk/ wcci2008/competitions.htm, accessed on November 17, 2008.

5. J. D. Knowles and D. W. Corne, The Pareto Archived Evolution Strategy: A New Baseline Algorithm for Pareto Multiobjective Optimization, in Proc. 1999 Congress on Evolutionary Computation (Washington, DC, 1999), p. 98-105.

6. K. Deb and R. B. Agrawal, Simulated Binary Crossover for Continuous Search Space, Complex Systems, 9, p. 115-148 (1995).

7. K. Deb, Muiti-objective Optimization Using Evolutionary Algorithms (Wiley, New York, 2001).

8. M. Tomassini, Evolutionary Algorithms, in Proc. Int. Workshop on Towards Evolvable Hardware, the Evolutionary Engineering Approach, eds. E. Sanchez and M. Tomassini (Switzerland, Lausanne, 1995), p. 19-47. 